\title{
Lumen
}

Selected Proceedings from the Canadian Society for Eighteenth-Century Studies

\section{Existence et temporalité au Siècle des lumières. Turgot lecteur de Maupertuis et Berkeley}

\section{Sébastien Charles}

Volume 21, 2002

URI : https://id.erudit.org/iderudit/1012267ar

DOI : https://doi.org/10.7202/1012267ar

Aller au sommaire du numéro

Éditeur(s)

Canadian Society for Eighteenth-Century Studies / Société canadienne d'étude du dix-huitième siècle

ISSN

1209-3696 (imprimé)

1927-8284 (numérique)

Découvrir la revue

Citer cet article

Charles, S. (2002). Existence et temporalité au Siècle des lumières. Turgot

lecteur de Maupertuis et Berkeley. Lumen, 21, 45-59.

https://doi.org/10.7202/1012267ar

Copyright (C Canadian Society for Eighteenth-Century Studies / Sociéte canadienne d'étude du dix-huitième siècle, 2002
Ce document est protégé par la loi sur le droit d'auteur. L'utilisation des services d'Érudit (y compris la reproduction) est assujettie à sa politique d'utilisation que vous pouvez consulter en ligne.

https://apropos.erudit.org/fr/usagers/politique-dutilisation/ 


\section{Existence et temporalité au Siècle des lumières. Turgot lecteur de Maupertuis et Berkeley}

Le portrait que l'on trace des Lumières est essentiellement à l'avantage de Locke sur Descartes. Rien de plus exact en termes d'influence. Pourtant, si tous les penseurs du XVIII ${ }^{\mathrm{e}}$ siècle semblent partager une vision empiriste du monde, il n'en reste pas moins que certains problèmes légués par le dualisme ontologique cartésien continuent de se poser, quoique dans des termes différents. Ainsi, bien que confiant dans les potentialités de la raison, dans le progrès des connaissances et dans la perfectibilité morale et politique des sociétés humaines, ce siècle n'en est pas moins, au plan épistémologique, une époque fortement marquée par des considérations sceptiques. Giorgio Tonelli a bien montré, dans un article célèbre paru en 1971 et réédité récemment ${ }^{1}$, que l'optimisme des Lumières ne pouvait être réellement compris sans tenir compte de sa contrepartie sceptique et de sa velléité à limiter les prétentions de la raison, notamment dans le domaine métaphysique. D'où une critique radicale à l'égard de toute volonté d'outrepasser les bornes naturelles de la raison, et un doute général développé envers les notions de système, de substance, d'infini, de transcendance, etc., la finalité d'un tel processus étant bien sûr de séparer radicalement le domaine de la foi de celui de la raison, la théologie de la philosophie, séparation qui n'avait été qu'envisagée par le siècle précédent.

Pour notre part, nous ne nous intéresserons ici qu'à un des problèmes mis en avant par Tonelli, à savoir la critique de la notion de substance, que nous évoquerons à travers la question de l'existence des corps extérieurs qui vit s'opposer le réalisme de Turgot, partisan farouche de l'existence d'une substance rendant compte des phénomènes, au

1 Giorgio Tonelli, "The "Weakness" of Reason in the Age of Enlightenment», dans R. H. Popkin, E. de Olaso et G. Tonelli (édit.), Scepticism in the Enlightenment, Dordrecht, Kluwer, 1997, p. 35-50. 
phénoménisme de Maupertuis et de Berkeley. Cette controverse sur le fondement de l'existence du monde extérieur est également, on le verra, une controverse sur le rapport de la substance au langage et à la temporalité.

À en croire son plus célèbre biographe, Condorcet, «M. Turgot disait souvent qu'un homme qui n'avait jamais regardé la question de l'existence des objets extérieurs comme un objet difficile et digne d'occuper notre curiosité, ne ferait jamais de progrès en métaphysique ${ }^{2} . »$ Et il faut dire que ce jugement était commun à l'époque. En témoigne le Projet d'un traité diderotien où, dans la partie «Les sciences», on peut lire: «La métaphysique. Berkeley ${ }^{3}$.» Il apparaît que, pour Diderot, une métaphysique qui n'aurait pas mis en question ses fondements à travers une confrontation critique avec Berkeley, et donc avec la question de l'existence du monde extérieur, ne vaudrait pas une heure de peine. Turgot en avait sans doute conscience, puisqu'il consacra dans sa jeunesse deux Lettres à l'abbé de ... sur le système de Berkeley ${ }^{4}$, que l'on peut dater de 1750 et dont la publication sera posthume, critique reprise par la suite dans l'article «Existence ${ }^{5}$ » de l'Encyclopédie qui aurait dû avoir pour conclusion logique, selon Turgot, un article sur l'«Immatérialité» qu'il ne fournira jamais à une entreprise (l'Encyclopédie) désormais con-

2 Condorcet, CEuvres de Condorcet, éd. O'Connor-Arago, Paris, Didot, 1847, vol. V, p. 172 , note 1 . L'importance de ce problème pour Turgot n'a pas attiré l'attention des ouvrages consacrés à sa pensée. Si certains commentateurs font de la réfutation de l'immatérialisme un appendice de sa métaphysique, d'autres vont jusqu'à ne pas la mentionner, alors qu'ils prétendent donner un panorama complet de la philosophie de ce dernier. C'est le cas d'Antonio Lenarda (auteur d'un «Turgot filosofo», dans L. S. Olschki (édit.), Atti e memorie dell'Academia toscana di scienze e lettera La Colombiara, 41, 1976, p. 213-271) qui ne voit en Turgot qu'un penseur des notions de "progrès» et de "perfectibilité». Sur ce point, nous rejoignons la critique similaire de Bruno Miglio, «Studi recenti su Turgot», Rivista di filosofia, 69, 1978, p. 353. En France, l'ouvrage de Claude Morilhat (la Prise de conscience du capitalisme. Économie et philosophiechez Turgot, Paris, Méridiens Klincksieck, 1988) sacrifie malheureusement le philosophe à l'économiste.

3 Denis Diderot, CEuvres complètes, éd. Lewinter, Paris, Le Club du livre, 1972, vol. XIII, p. 902.

4 Dans Gustave Schelle (édit.), CEuvres de Turgot et documents le concernant, avec biographie et notes, Paris, Alcan, 1913, vol. I, p. 185-193. Nous citerons désormais les textes de Turgot dans cette édition, qui est celle de référence. Sur la lecture critique de Berkeley par Turgot, voir notre «Turgot ou la pensée fragmentée. Étude d'une critique de l'immatérialisme berkeleyen", Études maritainiennes, 15, 1999, p. 157-167.

5 CEuvres de Turgot, vol. I, p. 517-538. 
damnée par le gouvernement et qui prenait peu à peu, à ses yeux, l'allure d'une «secte ${ }^{6}$ ».

Pour bien comprendre l'opposition de Turgot aux thèses immatérialistes, il faut étudier non seulement les textes où il s'oppose nommément à Berkeley, mais également sa réfutation des Réflexions philosophiques sur l'origine des langues et la signification des mots de Maupertuis. Dans cet ouvrage, qui est sans doute le plus proche des thèses berkeleyennes, Maupertuis s'en prend explicitement au concept cartésien d'étendue. Pour lui, Descartes a réduit la matière à l'étendue tout simplement parce qu'il a cru reconnaître, grâce à la vue et au toucher, son existence présente dans tous les corps. Mais si une autre qualité sensible, appartenant à un registre sensoriel différent (l'odorat, par exemple, et non le toucher), avait été présente uniformément au sein de tout l'univers, si toute chose avait été ainsi odeur de rose, n'aurions-nous pas dû pour autant en conclure logiquement que l'odeur de rose était la substance des choses? C'est la question que pose Maupertuis en prenant l'exemple, cette fois, de la couleur: «Je voudrais bien qu'on examinât si, en cas que tous les objets du Monde fussent verts, on n'aurait pas eu la même raison de prendre la verdeur pour substance ${ }^{7} . »$ Pour le dire brièvement, Maupertuis remet ici radicalement en question l'avantage que certains philosophes ont attribué à une qualité du réel sur les autres:

Si la perception que j'ai d'arbre est bien fixée et limitée, on ne saurait en rien retrancher sans la détruire. Si elle n'est composée que d'étendue, figure et verdeur, et que je la dépouille de verdeur et figure, il ne restera qu'une perception vague d'étendue. Mais n'aurais-je pas pu par de semblables abstractions dépouiller l'arbre de l'étendue et de la figure, et ne serait-il pas resté tout de même une idée vague de verdeur?

6 C'est la thèse de Gerald J. Cavanaugh défendue dans «Turgot and the Encyclopédie», Diderot Studies, 10, 1968, p. 27 et 29-30, qui est sans doute vraisemblable, même si d'autres aspects ont pu jouer. L'article «Immatérialisme» de l'Encyclopédie est, selon Pierre Rétat (le Dictionnaire de Bayle et la lutte philosophique au XVIII siècle, Lyon, Audin, 1971, p. 404), attribuable à l'abbé Yvon et non à Turgot.

7 Maupertuis, Réflexions sur l'origine des langues, et sur la signification des mots, dans CEuvres de Maupertuis, Lyon, Jean-Marie Bruyset, 1768, vol. I, p. 272. Nous citerons désormais les textes de Maupertuis dans cette édition dont nous avons modernisé l'orthographe. Sauf indication contraire, tous les italiques proviennent du texte original.

8 Ibid. 
D'où le refus net, chez Maupertuis, de voir en l'étendue la substance des êtres, refus qui, dit-il, est partagé par le vulgaire. Cette intuition est proche de ce que pense Berkeley, plus sage sur ce point que les philosophes de son temps, qui sont incapables de s'entendre sur ce que signifient les termes de substance et de mode.

Cette argumentation paraît intenable à Turgot qui pense que Berkeley et Maupertuis mènent un combat identique, quoique perdu d'avance, en faveur du phénoménisme. Pour Turgot, en effet, toute doctrine phénoméniste ne peut être qu'erronée, puisque les objets existent indépendamment des perceptions que nous en prenons, ce qui indique que certaines qualités leur sont propres, et cela même si elles nous restent inconnaissables parce qu'imperceptibles. La fausseté des philosophies de Maupertuis et de Berkeley tient à deux erreurs fondamentales que Turgot n'a de cesse de dénoncer, qui se situent au plan du langage et de la temporalité.

En ce qui concerne le langage, Maupertuis et Berkeley ont accordé trop d'importance à la thèse moderne de l'hétérogénéité des sensations, en pensant que chaque terme avait pour cause de son origine linguistique les sensations que nous en prenions, ce qui amenait immanquablement à réduire sa signification à celles-ci. Turgot ne déforme pas ici la position de ses adversaires, puisque c'est explicitement ce que Berkeley tente de faire comprendre à Hylas par la bouche de Philonous en prenant l'exemple fameux entre tous de la cerise:

Je vois cette cerise, je la touche, je la goûte; je suis sûr que le néant ne peut être vu, être touché, être goûté; elle est donc réelle. Enlevez les sensations de mollesse, d'humidité, de rougeur, d'acidité, et vous enlevez la cerise [...]. En conséquence, quand je vois et que je touche et que je goûte en certaines diverses manières, je suis sûr que la cerise existe, qu'elle est réelle, puisque sa réalité n'est pas à mon avis quelque chose d'abstrait à séparer de ces sensations. Mais si vous entendez par le mot de cerise une nature inconnue distincte de toutes ces qualités sensibles, et par son existence quelque chose de distinct de ce qu'elle est perçue, alors, certes, je l'avoue, ni vous ni moi ni personne d'autre ne peut être assuré qu'elle existe?

9 Berkeley, Dialogues entre Hylas et Philonous, dans Luce et Jessop (édit.), The Works of George Berkeley Bishop of Cloyne (désormais Works), London, Nelson, 1956, vol. II, p. 249 (CEuvres, vol. II, p. 131). Nous citons en parallèle et entre parenthèses la référence à l'édition des CEuvres de Berkeley (désormais CEuvres), parue sous la direction de Geneviève Brykman, Paris, Presses universitaires de France, coll. «Épiméthée», 1985-1996, 4 vol. 
Pour Berkeley, on le voit, dissiper le voile des mots, c'est comprendre que leur signification se borne entièrement aux perceptions que nous avons des objets qui leur correspondent et qu'ils ne sauraient signifier autre chose, puisque l'être même des choses se réduit à leur apparaître.

Turgot, au contraire, pense que la dénomination va au-delà de la perception, ce qui l'amène à substantifier le langage en montrant qu'il vise l'essence des choses et non leurs accidents:

Le premier dessein du langage et le premier pas est d'exprimer les objets et non les perceptions; ce second dessein ne vient à l'esprit que lorsque, dans le sang-froid du retour sur soi-même, la perception elle-même devient un objet de perception. Cela paraîtra d'autant plus évident que nos premières idées sont des sensations et que, par l'effet naturel des sensations, nous les rapportons promptement aux objets extérieurs ${ }^{10}$.

L'unité des sensations est du côté de l'objet, non du sujet, et c'est cette unité objective que le mot désigne. Sinon, les choses se réduiraient à la perception que nous en prenons et l'être à l'apparaître. Or Turgot voit bien ici les dangers potentiels de toute position phénoméniste radicale, non seulement pour les choses perçues qui s'épuisent dans leur paraître, mais également pour l'esprit percevant. Refuser toute substance matérielle, c'est prêter le flanc à une radicalisation de la critique de la notion de substance, qui pourrait s'exercer par la suite à l'encontre de la substance pensante elle-même. En effet, si les choses n'ont pas besoin d'une substance pour se maintenir dans l'être, pourquoi les idées auraient-elles besoin d'un moi unificateur? Turgot sent bien, de manière intuitive, que Berkeley mène inexorablement à Hume. C'est pourquoi il lui paraît capital de sauver l'idée d'être, tant matériel que spirituel: «C'est l'idée d'être en général et non celle de substance qui répond à ce qu'il y a d'uniforme, non dans les perceptions, mais dans les objets; c'est l'idée de moi qui est la seule chose uniforme dans les perceptions ${ }^{11}$.» Sans cette uniformité objective et subjective, le solipsisme est la règle. En effet, si l'on considère les sensations comme indépendantes des objets qui en

10 Turgot, Remarques critiques sur les Réflexions philosophiques de Maupertuis sur l'origine des langues et la signification des mots (1750), dans CEuvres de Turgot, vol. I, p. 162. Voir aussi p. 173-174: «Maupertuis suppose partout que nous cherchons des mots pour nos perceptions. Au contraire, ce sont les choses que nous cherchons surtout à exprimer" (nous soulignons).

11 Ibid., p. 166-167. 
sont la marque, il est impossible de sortir de soi et de se prononcer sur aucune existence autre que la sienne.

Reste alors à postuler, sous une forme très traditionnelle ${ }^{12}$, que le réel est un ensemble d'êtres en relation causale les uns avec les autres, relation qui ne se réduit pas à une pure phénoménalité. Les choses possèdent bien une profondeur intrinsèque qui en fait des substances. C'est ce que Maupertuis et Berkeley, selon Turgot, n'ont pas compris, puisqu'ils confondent $u n e$ substance et la substance. Une substance, pour Turgot, c'est une somme de flux de perceptions qui existe dans la réalité: ce peut être un esprit ou un être sensible. Un arbre, en tant qu'on le considère dans son intégralité, est une substance, "car le mot de substance est un nom que les hommes ont donné à l'objet existant hors d'eux auquel se rapportent leurs différentes perceptions ${ }^{13}$ ". La substance, au contraire, est un mot vague dont l'étymologie nous rappelle la provenance. C'est parce qu'on a pensé la substance comme un substratum qu'on a été amené à la distinguer de ses modes ou qualités secondes qui ont un rapport nécessaire à l'esprit qui les perçoit. Dans ce second sens, la substance, $c^{\prime}$ est ce qui persiste à travers ses modifications. Mais quelle est-elle réellement?

Les Cartésiens, voyant qu'on ne pouvait dépouiller les corps de l'étendue en ont conclu que c'était en cela que consistait la substance des corps. Mais, est-ce l'étendue qui est la substance, ou n'est-elle pas elle-même le résultat de plusieurs substances, comme le veulent les Leibniziens? Et qui est-ce qui fait que les monades sont substances? C'est ce que nous ne pouvons savoir, sans connaître la nature des choses, dont, hélas!, nous ne connaissons que les rapports. Vouloir dire quelque chose de plus, c'est confondre les bornes de notre esprit et celles de la nature ${ }^{14}$.

C'est ici que se situe la limite actuelle de nos connaissances. Le tort de Maupertuis et de Berkeley, aux yeux de Turgot, est d'avoir voulu outrepasser les bornes de l'esprit humain en croyant pouvoir rejeter définitivement la substance étendue parce qu'ils ne pouvaient la comprendre.

12 Turgot reprend en effet en faveur de l'existence des corps extérieurs l'argumentation cartésienne du Dieu vérace, la notion d'inclination naturelle et le recours au consentement universel.

13 Turgot, Remarques critiques sur les Réflexions philosophiques de Maupertuis, dans CEuvres de Turgot, vol. I, p. 167.

14 Ibid., p. 168. 
Turgot est donc convaincu que l'impossibilité philosophique dans laquelle nous sommes de donner une définition précise de ce qu'est une substance n'a pas pour corollaire son inexistence. En effet, quoique les philosophes ne s'entendent pas sur ce qui fait la substance des choses (étendue, matière, monades, etc.), ils s'entendent tous sur le fait qu'il y a une substance des choses. D'ailleurs, comment admettre l'existence d'une telle notion, communément partagée dans le monde philosophique, si les mots ne reflètent que nos sensations? Comment cette notion aurait-elle pu venir aux esprits philosophiques si elle n'existait pas? L'explication la plus plausible revient à supposer son existence réelle, et ce même si les conditions de cette existence nous restent inconnues. Mais cet aveu d'impuissance est propédeutique et non définitif, toute la pensée de Turgot s'ordonnant autour des notions de "progrès» et de «perfectibilité ${ }^{15}{ }^{»}$ des connaissances, ce qui laisse à penser que l'avenir pourra peut-être pallier les errements épistémologiques de son siècle et apporter, là où il ne voit qu'une conjecture fort probable, une certitude inébranlable.

Que l'avenir lui donne ou non raison, Turgot n'en pense pas moins qu'au présent le fardeau de la preuve de l'existence du monde extérieur repose sur les épaules de ses adversaires. Car, à supposer que les choses ne soient rien d'autre que ce que nous en percevons, comment expliquer cette stabilité dans l'être que nous semblons voir exister en elles? Devons-nous supposer que les choses s'anéantissent dès que nous ne les percevons plus et retrouvent l'existence à notre seul contact perceptif? Cette critique est facilement résolue par Berkeley, elle l'est moins par Maupertuis. Dès ses notes de jeunesse, Berkeley a écrit, de manière quelque peu énigmatique, que, à supposer que personne ne les perçoive, «le cheval est dans l'écurie, les livres sont dans le cabinet de travail comme auparavant». Ce qui veut simplement dire que les choses ne dépendent pas de la perception que notre esprit en prend, même s'il leur est impossible d'exister sans être perçues par un esprit, celui de Dieu $^{16}$. Maupertuis, qui prétend se passer du recours divin, propose

15 Notion dont d'ailleurs Turgot est l'inventeur, comme le rappelle Simone Goyard-Fabre, «L'optimisme juridique au XVIII siècle: ses raisons, ses limites», De Philosophia, 13, 1, 1997, p. 59.

16 Berkeley, Dialogues entre Hylas et Philonous, dans CEuvres, vol. II, p. 108 (Works, vol. II, p. 230-231): «Quand je refuse aux choses sensibles une existence hors de l'esprit, je n'entends point parler de mon esprit en particulier, mais bien de tous les esprits. Or il est manifeste qu'elles ont une existence extérieure à mon esprit, puisque je découvre par expérience qu'elles sont indépendantes de lui. Il y a donc quelque autre esprit 
de son côté une hypothèse originale dans laquelle le temps joue un rôle fondamental.

Ce recours au temps comme garantie ontologique de l'indépendance du monde n'est pas chose nouvelle. Déjà, au début du XVIII ${ }^{e}$ siècle, alors même que la question du solipsisme commençait à apparaître, l'invocation de la temporalité paraissait témoigner en faveur de l'existence $\mathrm{d}$ 'autre chose que de l'ego. Le père Tournemine, directeur des Mémoires de Trévoux à l'époque, et sans nul doute, à travers ses deux recensions partiales des Principes de la connaissance humaine et des Dialogues entre Hylas et Philonous de Berkeley ${ }^{17}$, le grand responsable de l'amalgame entre immatérialisme et scepticisme, comprend bien qu'accepter la doctrine solipsiste, dont Berkeley lui parait être le champion, c'est miner l'une des assises fondamentales de la foi chrétienne, à savoir la preuve de l'existence de Dieu a contingentia mundi. Dès lors, il faut réfuter cette philosophie nouvelle, et, pour ce faire, Tournemine n'a pas peur de la répétition. Il va donc faire paraître trois fois, entre 1713 et 1719 , des réflexions sur l'athéisme qui s'en prendront explicitement aux partisans de Berkeley ${ }^{18}$. Pourquoi tant d'acharnement? Simplement parce que Tournemine croit que l'immatérialisme est en train de prendre le pas sur le spinozisme et séduit des couches de plus en plus larges de l'intelligentsia parisienne. Le meneur de cette secte nouvelle, Berkeley, semble prendre le contre-pied exact du spinozisme, du moins de ce que Tournemine pense être le spinozisme, en affirmant que tout est esprit, là où Spinoza disait que tout était matière. Ce qui revient, on s'en doute, à prôner un athéisme tout aussi radical qui renverse toute preuve cosmologique de l'existence de Dieu:

dans lequel elles existent, durant les intervalles entre les moments où je les perçois; comme aussi bien elles l'ont fait avant ma naissance et le feraient après mon anéantissement supposé. Et puisque cette vérité vaut identiquement au regard de tous les autres êtres créés et finis, il s'ensuit nécessairement qu'il y a un Esprit éternel omniprésent, qui connaît et comprend toutes choses, et qui nous les donne à voir de la manière et conformément aux règles qu'il a lui-même prescrites, et que nous appelons les lois de la nature."

17 Parus respectivement dans les Mémoires de Trévoux en mai 1713, p. 921-922, et en décembre 1713, p. 2198-2199. Sur cette première réception de Berkeley par les hommes $\mathrm{du}$ XVIII $^{\mathrm{e}}$ siècle, voir notre "Deux cas de réception biaisée au siècle des Lumières: Spinoza et Berkeley", Actes du XIV Congrès interaméricain de philosophie, CD-ROM, Puebla, 2000, p. 598-611.

18 Tournemine, «Réflexions sur l'athéisme», dans Fénelon, CEuvres philosophiques, Paris, Estienne, 1713, rééditées en 1718 et de nouveau publiées, séparément, dans les Mémoires de Trévoux de janvier 1719, p. 14-54. 
l'impiété se flatte de s'être par ce nouveau système délivré de tout devoir et de tout remords; d'avoir ôté aux vrais Philosophes tous les moyens de prouver l'existence de Dieu, de s'être mise enfin dans un repos que rien ne peut troubler. On pressait les impies par la démonstration tirée de la structure de chaque corps en particulier, et de la construction entière du Monde sensible, ils l'anéantissent, c'est la plus courte voie pour se débarrasser de la démonstration. Dans leur système point de liaison entre les êtres pensants, chacun est un monde indépendant des autres ${ }^{19}$.

Heureusement, Tournemine en est persuadé, ce système, par ses excès mêmes, n'aura guère de sectateurs. D'une part, parce qu'il est opposé au sens commun; d'autre part, parce que la présence en nous d'idées qui ne sont pas de nous implique l'existence d'un Dieu tout-puissant (mais cela, et Tournemine ne le voit pas, ne permet pas de conclure que le monde a été créé); et enfin parce que tout immatérialiste conséquent doit ou reconnaître que son esprit a été créé (ce qui suppose de remonter à une première cause, ce que les athées refusent) ou qu'il est éternel, mais, en ce cas, il est un moment où il ne pensait pas et il n'est actif que depuis un certain temps, ce qui implique de faire intervenir la temporalité au sein même de l'éternité, ce qui est absurde. Bref, Tournemine conclut en montrant qu'il s'agit là de "suppositions bizarres dont on ne voit aucun fondement; en vérité les incrédules voudraient nous faire croire des choses plus incroyables que tous les mystères ${ }^{20}{ }$.

Tournemine est ainsi le premier à se servir de la notion de temporalité pour résoudre l'aporie solipsiste. Mais cette justification suffit-elle? Un autre Jésuite, le père Buffier, va répondre par la négative. Au problème posé par Tournemine - l'immatérialiste doit reconnaître que son esprit a été créé (ce qui implique que Dieu existe et donc qu'il n'est pas le seul être existant) ou qu'il est éternel, mais alors comment expliquer qu'il ne pense pas toujours et qu'il n'a pas toujours pensé? -, Buffier fournit une réponse convaincante. Elle consiste à pousser le solipsisme à son terme, en montrant que la perception qui rend évident le sentiment intime de l'existence propre d'un solipsiste se doit d'être toujours actuelle, c'est-àdire vécue au présent, le souvenir n'existant que s'il est perçu hic et nunc, ce qui explique que le passé n'a de sens qu'au présent. Or, parce que la mémoire est faillible et finie, elle ne peut rendre compte que d'une partie

19 Tournemine, «Réflexions sur l'athéisme», dans Fénelon, OEuvres philosophiques (1718), Amsterdam, Zacharie Chatelain, 1731, p. 364-365.

20 Ibid., p. 368. 
de l'existence et non de sa totalité. Dès lors, tout ce qui porte sur le passé est sujet à caution:

Chacun de nous doit être persuadé qu'il n'a aucune certitude évidente s'il n'a point éternellement subsisté; puisqu'il pourrait avoir subsisté de la sorte sans qu'il s'en ressouvienne. Que si on lui représente qu'il a été produit, il pourra répondre qu'il n'en a point de certitude évidente. Car avoir été produit est une chose passée, et n'est pas la perception ni le sentiment intime de ce qui se passe actuellement en nous ${ }^{21}$.

Il semble donc que le solipsisme soit irréfutable une fois posé le sentiment intime.

Peut-on réellement sortir de soi quand on ne reconnaît comme seule évidence que le sentiment intime que l'on prend de son existence propre? Cette question n'est-elle pas encore plus délicate pour les partisans du sensualisme, qui paraissent incapables d'aller au-delà de leur propre sensibilité? A son tour, Maupertuis va tenter de régler cette problématique, en faisant lui aussi intervenir la temporalité comme garantie ontologique de l'existence du monde. Car, si toute la théorie des idéessignes maupertuisienne repose sur les sens et s'ordonne autour des perceptions qu'ils reçoivent de l'extérieur et qui renvoient à des choses externes mais non substantielles, elle ne saurait se passer de leur dimension temporelle. En effet, les perceptions sensibles peuvent être étagées selon leur vivacité et en fonction du travail de la mémoire - une perception présente sera alors plus marquée qu'une perception passée, et un élément réel plus vivace qu'un élément rêvé (Berkeley ne dit pas autre chose) - , mais entre également en ligne de compte la notion de répétition expérientielle. Ce qui fonde dans l'être les choses corporelles, c'est leur permanence dans le temps aperçue par le biais de cette répétition temporelle:

J'ai la perception j'ai vu un arbre, jointe à la perception j'étais dans un certain lieu; j'ai celle j'ai retourné dans ce lieu, j'ai vu cet arbre; j'ai retourné encore dans le même lieu, j'ai vu le même arbre, etc. Cette répétition, et les circonstances qui l'accom-

21 Buffier, Traité des premières vérités, et de la source de nos jugements, part. I, chap. 2 (1724), repris dans Cours de science sur des principes nouveaux et simples; pour former le langage, l'esprit et le aeur, dans l'usage ordinaire de la vie, Paris, Guillaume Cavelier et Pierre-François Giffart, 1732, p. 561-562. 
pagnent, forment une nouvelle perception, je verrai un arbre toutes les fois que j'irai dans ce lieu; enfin il y a un arbre $e^{22}$.

À partir de là, selon Maupertuis, cet arbre peut être conçu comme indépendant de l'individu qui le perçoit et il n'a pas besoin d'être actuellement perçu pour être dit existant. Maupertuis suppose donc une certaine stabilité du paraître, soumis à des lois naturelles qui lui permettent de perdurer dans le temps indépendamment dé la perception que les esprits peuvent en prendre. Le temps est un facteur de stabilité ontologique, il donne une consistance réelle aux objets que l'on doit supposer toujours existant à une même place, et ce même s'ils ne sont pas ou plus perçus. Cela étant, Maupertuis ne se pose pas vraiment la question de savoir ce que devient l'arbre quand il n'est pas perçu, supposant simplement une stabilité temporelle dans l'être hors de toute perception (la différence avec Berkeley est ici patente, pour qui esse est percipi, et chez qui Dieu est l'armature ontologique du monde garantissant sa permanence dans l'être). Cette hypothèse quelque peu étrange d'une temporalité vue comme garantie ontologique ne paraît pourtant pas suffisante à Maupertuis, car la durée se réduit elle aussi à une perception subjective, et le passé à notre appréhension mentale actuelle. Le monde ne serait-il alors que le produit de notre conscience, comme le supposaient les égoïstes du début du siècle, se croyant seuls réels? Le fait qu'il n'apparaisse pas décousu et qu'il semble manifester une certaine harmonie permet d'en douter. Mais comment expliquer ce phénomène qui veut que la succession de nos perceptions se fasse selon un certain ordre et un certain rapport? Et comment une perception psychique (une idée) peut-elle être confirmée dans le temps par une perception physique (la vue d'un objet)? Pourquoi est-on sûr en ayant la perception d'aller dans un endroit où on a vu un arbre qu'on aura par la suite celle de voir réellement cet arbre?

Toutes ces questions lui valent les sarcasmes de Turgot: "Quant à la raison pourquoi l'idée, je vois un arbre, succède à celui-ci [sic], je vais dans un endroit où j'ai vu un arbre, elle est simple, c'est que l'arbre y est et Maupertuis me fait rire ${ }^{23}$.» Le temps n'accorde pas aux êtres leur permanence; au contraire, celle-ci leur est intrinsèque, car ce sont des substances:

22 Maupertuis, Réflexions sur l'origine des langues et la signification des mots, p. 279.

23 Turgot, Remarques critiques sur les Réflexions philosophiques de Maupertuis sur l'origine des langues et la signification des mots, dans CEuvres de Turgot1, vol. I, p. 178. 
Pourquoi éprouve-t-on une série successive d'impressions semblables, quand on va plusieurs fois dans le même lieu? La réponse est bien simple, c'est que les mêmes objets s'y retrouvent, et que ces objets étant la cause de nos impressions, les mêmes causes produisent nécessairement les mêmes effets sur notre sensibilité, qui reste aussi la même. Mais si nous nions l'existence des objets extérieurs, il est impossible de saisir la raison de cette série d'impressions semblables ${ }^{24}$.

Voilà sans doute pourquoi Turgot prévoyait être l'auteur de l'article «Mémoire» dans l'Encyclopédie, car seule cette faculté, selon lui, permet d'attribuer l'être aux apparences sensibles qui affectent nos sens. Si cela est le cas, Turgot ne prend-il pas à son tour la même voie que Maupertuis et ne s'est-il pas un peu vite moqué de ce dernier? Que la permanence soit ou non un phénomène intrinsèque aux choses, il n'en reste pas moins que la temporalité est, dans les deux cas, indispensable à la manifestation de leur stabilité. Le temps ne permet pas de départager réalisme et phénoménisme. Seule une preuve concluante en faveur de l'existence des corps extérieurs compris en tant que substance et non en tant qu'apparences perceptives pourrait trancher définitivement la question.

Turgot se voit donc contraint de prouver que les corps extérieurs ne se réduisent pas aux sensations que nous en prenons et que leurs qualités premières (objectives) ne sont pas réductibles à leurs qualités secondes (subjectives). Cela l'amène tout naturellement à rompre avec la doctrine de l'hétérogénéité des sensations. Alors que Maupertuis et Berkeley avançaient l'hypothèse d'une combinaison des sensations par l'être percevant, le moi ou l'esprit, Turgot veut que cette combinaison ait lieu du côté de l'objet. C'est pourquoi un objet vu et touché en même temps est "cause commune de mes deux sensations ${ }^{25}$ ", ce qui implique une irréductibilité certaine. En effet, le double contact garantit la permanence dans l'être et la vraisemblance des perceptions. Alors que, chez Descartes, c'est le jugement qui «redresse» le bâton tordu dans l'eau, chez Turgot, c'est un sens qui fonde la certitude des autres. Il peut certes tromper, mais l'union perceptive de plusieurs sens est gage de réalité objective.

Outre l'hétérogénéité des sensations, Turgot se sert de la dualité ontologique existant entre être et apparaître pour convaincre ses adversaires. L'ordre perceptif et l'ordre réel ne sauraient être identiques,

24 Alfred Mastier, Turgot, sa vie et sa doctrine, Paris, Guillaumin et Durand, 1862, p. 188.

25 Première lettre à l'abbé de... sur le système de Berkeley, dans CEuvres de Turgot, vol. I, p. 186. 
puisque certaines choses existent alors même que nous n'en avons aucune perception:

Puisque Rome et Londres existent pour nous lorsque nous sommes à Paris, puisque nous jugeons leur être existant indépendamment de nos sensations et de notre propre existence, l'ordre de nos sensations qui se présentent à nous les unes hors des autres, et l'ordre des êtres placés dans l'espace à des distances réelles les unes des autres, forment donc deux ordres de choses, deux mondes séparés, dont un au moins (c'est l'ordre réel) est absolument indépendant de $l^{\prime}$ autre ${ }^{26}$.

Le monde est insensé si nous sommes à jamais enfermés dans notre moi, si les choses extérieures ne sont qu'une modification de notre sensibilité et ne renvoient pas à un monde réel. Quelle serait la finalité d'une nature tissée uniquement d'apparences? Quel avantage pourrait tirer une divinité supposée bonne de l'existence non réelle de ce qui nous affecte? Pourquoi nous faire hurler de douleur si le feu qui nous brûle n'est qu'une modification perceptive et donc, en dernier lieu, un phénomène irréel? Un Dieu sage et bon le permettrait-il? En un mot, nous dit Turgot, «tout est expliqué en supposant l'existence des corps; tout est obscur et bizarre - en la niant ${ }^{27}{ }^{»}$. Mais invoquer la bonté divine ne saurait suffire, et ce même si Turgot semble parfois s'en contenter ${ }^{28}$.

Reste à trouver un principe inébranlable, et c'est celui de causalité ou $d^{\prime}$ induction, qui consiste à partir des effets pour remonter à leur cause. «L'argumentation de Turgot repose sur un syllogisme que l'on pourrait résumer ainsi: il n'y a pas d'effets sans causes, or les sensations sont des effets, elles ont donc des causes ${ }^{29} .{ }^{\prime}$ Dès lors, le monde extérieur lui semble recouvrer toute sa profondeur et sa richesse, et le moi qui s'en croyait seul créateur devient une infime partie d'un tout qui existe objectivement.

26 Article «Existence», dans CEuvres de Turgot, vol. I, p. 532-533.

27 Deuxième lettre à l'abbé de... sur le système de Berkeley, dans CEuvres de Turgot, vol. I, p. 190.

28 Voir, par exemple, ce passage significatif de la Deuxième lettre, ibid.: "Combien de sensations désagréables surtout qui nous avertissent des dangers de notre corps, et qui ne seraient de la part de Dieu qu'un jeu cruel, si les corps n'existaient pas! Voilà donc les objets extérieurs démontrés à nous existants" (nous soulignons).

29 Jacques Wendel, "Turgot et l'Encyclopédie», thèse de doctorat, Ohio State University, 1978, p. 182. 
Il est vrai que ce système est toujours lié avec nous, par la conscience de nos pensées présentes; mais il n'en est pas moins vrai que nous n'en sommes pas parties essentielles, qu'il existait avant nous, qu'il existera encore après nous, et que, par conséquent, le rapport qu'il a avec nous n'est pas nécessaire pour qu'il existe, et l'est seulement pour que son existence nous soit connue ${ }^{30}$.

C'est distinguer ici un monde en soi d'un monde pour nous, des choses en soi de choses perçues. Seule cette distinction, non effectuée par Maupertuis et Berkeley selon Turgot, permet de comprendre comment des phénomènes non perçus existent bel et bien, et même à l'intérieur de notre propre corps. Et pour ceux qui ne seraient pas convaincus par cet argument fondé sur la notion de causalité, il reste à Turgot la possibilité de pousser le principe qui lie existence et perception jusque dans ses conséquences les plus absurdes:

Je conçois bien que j'ai besoin, si je mange, d'un estomac et de viscères pour digérer: mais si je ne mange pas, ou si je ne mange qu'en idée, si mon estomac, que je ne vois point, $n^{\prime} e s t$ rien, pourquoi celui qui ouvrira mon corps aurait-il cette idée d'un estomac aussi peu utile pour lui que pour moi ${ }^{31}$ ?

Ce recours à la raillerie témoigne bien de l'impossibilité, pour Turgot, de réfuter définitivement le phénoménisme sur un plan argumentatif. Dire que les sensations sont des effets d'une cause qui nous reste cachée, $c^{\prime}$ est à la fois énoncer une proposition infalsifiable et ne pas tenir compte $\mathrm{d}$ 'une dimension importante de l'expérience humaine, celle du songe. Les critiques apportées à l'encontre des philosophies de Maupertuis et de Berkeley manquent leur cible et ne sont valides que lorsqu'elles réduisent le phénoménisme à un pur et simple solipsisme. Turgot dresse dès lors un faux procès en lisant en elles un approfondissement $\mathrm{du}$ sixième éclaircissement de la Recherche de la vérité de Malebranche, qui énonce que, sans la foi, l'existence du monde extérieur est improuvable ${ }^{32}$. Mais l'intérêt d'une telle confrontation entre réalisme et phénoménisme n'en reste pas moins entier. A travers ce débat, les philosophes des

30 Article «Existence», dans OEuvres de Turgot, vol. I, p. 530.

31 Deuxième lettre, dans CEuvres de Turgot, vol. I, p. 189-190. Le même argument est utilisé dans la Première lettre, ibid., vol. I, p. 187.

32 Nicolas Malebranche, Recherche de la vérité, Sixième éclaircissement, dans OEuvres complètes, édité par André Robinet, Paris, Vrin 1958-1967; ici vol. III édité par Geneviève Rodis-Lewis, 1964, p. 53-66. 
Lumières ont, une fois encore, ouvert et balisé la route qui mène à Kant, en comprenant que toute pensée de l'être est indissociablement une pensée du temps.

\section{SÉBASTIEN CHARLES}

Université de Sherbrooke 